\title{
Outcomes of HER2-positive early breast cancer patients in the pre-trastuzumab and trastuzumab eras: a real-world multicenter observational analysis. The RETROHER study
}

\author{
Patrizia Vici - Laura Pizzuti - Clara Natoli • Luca Moscetti - Lucia Mentuccia • \\ Angela Vaccaro $\cdot$ Domenico Sergi $\cdot$ Luigi Di Lauro $\cdot$ Patrizia Trenta \\ Patrizia Seminara $\cdot$ Daniele Santini $\cdot$ Laura Iezzi $\cdot$ Nicola Tinari $\cdot$ \\ Ilaria Bertolini · Valentina Sini · Marcella Mottolese · Diana Giannarelli • \\ Francesco Giotta $\cdot$ Marcello Maugeri-Saccà $\cdot$ Maddalena Barba $\cdot$ \\ Paolo Marchetti · Andrea Michelotti · Isabella Sperduti · Teresa Gamucci
}

Received: 1 August 2014/ Accepted: 9 September 2014/Published online: 19 September 2014 (C) The Author(s) 2014. This article is published with open access at Springerlink.com

\begin{abstract}
Addition of trastuzumab to adjuvant chemotherapy has dramatically reduced the risk of recurrence and has become the standard of care for human epidermal growth factor receptor 2 (HER2)-positive early breast cancer patients. Since most data on trastuzumab benefits come from clinical trials, conducted in selected patient populations, we performed a retrospective analysis of HER2-positive early breast cancer patients treated in the "pre-trastuzumab" and "trastuzumab" eras, with the aim
\end{abstract}

Patrizia Vici and Laura Pizzuti have contributed equally to this work and serve as first co-authors

P. Vici $(\bowtie) \cdot$ L. Pizzuti · D. Sergi · L. Di Lauro ·

M. Maugeri-Saccà · M. Barba

Division of Medical Oncology B, "Regina Elena" National

Cancer Institute, Via Elio Chianesi, 53, 00144 Rome, Italy

e-mail: pvici@ifo.it

C. Natoli $\cdot$ L. Iezzi $\cdot$ N. Tinari

Department of Experimental and Clinical Sciences, University

"G. d'Annunzio", Chieti, Italy

L. Moscetti

Division of Medical Oncology, Department of Oncology,

Belcolle Hospital, ASL Viterbo, Viterbo, Italy

L. Mentuccia · A. Vaccaro - T. Gamucci

Medical Oncology Unit ASL Frosinone, Frosinone, Italy

P. Trenta

Department of Radiology, Oncology and Human Pathology,

"Sapienza" University of Rome, Rome, Italy

P. Seminara

Oncology A Unit, Department of Internal Medicine, "Sapienza"

University of Rome, Rome, Italy to determine patients' outcomes in real-world practice. 925 consecutive HER2-positive breast cancer patients treated with adjuvant chemotherapy in ten Italian oncologic centers were identified. Patients who had received adjuvant chemotherapy alone (cohort A, 352 patients), and patients who had received adjuvant chemotherapy followed or combined with trastuzumab (cohort B, 573 patients) were analyzed. Relapse rate at 3 years, relapse-free survival, and overall survival were significantly more unfavorable in the cohort A than in the cohort B $(p<0.0001)$. In multivariate analysis, factors related to relapse were younger age, advanced stage at diagnosis, absence of hormonal and of trastuzumab therapy. The benefit derived from the addition

D. Santini

Department of Medical Oncology, University Campus Bio-

Medico, Rome, Italy

I. Bertolini · A. Michelotti

Oncology Unit I, Azienda Ospedaliera Universitaria Pisana, Pisa, Italy

V. Sini · P. Marchetti

Oncology Unit, Sant'Andrea Hospital, "Sapienza" University of Rome, Rome, Italy

M. Mottolese

Department of Pathology, "Regina Elena" National Cancer Institute, Rome, Italy

D. Giannarelli · I. Sperduti

Biostatistics Unit, "Regina Elena" National Cancer Institute, Rome, Italy

F. Giotta

Division of Medical Oncology, IRCCS, Giovanni Paolo II

Hospital, Bari, Italy 
of trastuzumab was independent of nodal status and hormonal receptors expression. A subgroup analysis including 163 "triple positive" tumors with high levels of estrogen and progesterone receptor (TP50) suggested that addition of trastuzumab to adjuvant chemotherapy and hormonal therapy did not translate into better outcomes. In our analysis, trastuzumab benefit was confirmed in all but a small subset of TP50 tumors subgroups. In this subset further investigations are needed.

Keywords HER2-positive · Adjuvant chemotherapy · Trastuzumab · Triple positive tumors - Breast cancer

\section{Introduction}

Breast cancer is a heterogeneous disease. Overexpression or amplification of HER2 is detectable in 15-25\% of tumors and is associated with poor prognosis $[1,2]$.

With the exception of endocrine therapy, no other systemic treatments like trastuzumab, a monoclonal antibody against the extracellular domain of HER2, demonstrated such a magnitude of beneficial effects. In the metastatic setting, the combination of chemotherapy and trastuzumab increased time to progression and overall survival (OS) [3]. Several randomized trials demonstrated the impact of adjuvant trastuzumab on relapse-free survival (RFS) and OS in early breast cancer patients with HER2-positive tumors [4-10], but few data are available on patients treated in routine practice outside of clinical trials. This issue was recently addressed by the GHEA study, which retrieved medical records of 1,002 patients treated in routine clinical practice with adjuvant chemotherapy followed by trastuzumab in 42 Italian oncologic centers [11]. A number of retrospective evaluations confirmed the unfavorable outcome of HER2-positive patients in the "pretrastuzumab" era, or the prognostic advantage of trastuzumab administration, even outside of clinical trials but, usually, data come from population-based studies, low number of patients, and short follow-up.

To this end, we carried out a multicenter, retrospective analysis of HER2 positive early breast cancer patients treated with adjuvant chemotherapy alone or with chemotherapy and trastuzumab in real-world practice, to determine patient's outcomes in terms of relapse rate at 3 years (RR-3 year), RFS, breast cancer specific survival (BCSS) and OS, and to investigate on the determinants of clinical outcomes.

M. Maugeri-Saccà · M. Barba Scientific Direction, "Regina Elena" National Cancer Institute, Rome, Italy

\section{Patients and methods}

Our cohort included HER2-positive, consecutive early breast cancer patients treated in adjuvant setting in routine practice in ten Italian oncologic centers from January 1998 to December 2011. Medical records related to demographic, clinical, pathologic, and molecular features, adjuvant therapies and outcomes were retrieved. Anonymized data were entered into a database. Two cohorts were analyzed: patients who received adjuvant chemotherapy without trastuzumab, mostly until 2005 (cohort A), and patients who received adjuvant chemotherapy followed by or combined with trastuzumab (cohort B), starting from 2006, when trastuzumab was approved in Italy as adjuvant treatment. Endocrine treatment and radiotherapy were given whenever indicated.

Pathology assessment was performed on surgical specimens by pathologists of participating centers. Estrogen receptors (ER) and progesterone receptors (PgR) status was considered positive if $\geq 1 \%$ of tumor cells were stained by mAbs 6F11 and 1A6 (Menarini), respectively. Ki-67 was tested using the mAb MIB1 (Dako) and regarded high if $\geq 14 \%$ of the cell nuclei were immunostained. HER2 overexpression was tested using the polyclonal antibody A0485 (Dako), and was considered positive if grade 3+ staining intensity by immunohistochemistry, or grade $2+$ with gene amplification by fluorescence, silver or chromogenic in situ hybridization was detected. If missing, the molecular features were centrally evaluated on formalinfixed, paraffin-embedded tissue sections, whenever available. Local institutional ethic committees approved this study.

\section{Statistical analysis}

The associations between variables were tested by Pearson Chi Square test or Fisher Exact test, when appropriate. The Hazard Ratio and confidence limits (CI) were estimated for each variable using the Cox univariate model. Significance was defined at the $p \leq 0.05$ level. A multivariate Cox hazard model was developed using stepwise regression (forward selection) by selecting significant variables upon univariate analysis. Enter limit and remove limit were $p=0.10$ and $p=0.15$, respectively. Survival was calculated by the Kaplan-Meier analysis from the date of surgery until the time of death for breast cancer (BCSS), death for any causes (OS), relapse (RFS), or last visit (OS and RFS). Survival curves were truncated at that time-point where the recommendations according to Pocock et al. were satisfied [12]. The log-rank test was used to assess differences between subgroups. Significance was defined at the $p \leq 0.05$ level. 
Table 1 Main baseline patient characteristics in 925 patients

\begin{tabular}{|c|c|c|}
\hline \multirow[t]{2}{*}{ Characteristic } & \multicolumn{2}{|l|}{$N(\%)$} \\
\hline & Cohort A (352 pts) & Cohort B (573 pts) \\
\hline
\end{tabular}

Age

\section{Median}

Range

Menopausal status

$$
\text { Pre }
$$

Post

Histology

Ductal

Lobular

Other

Tumor size

$\mathrm{T} 1$

$\mathrm{T} 2$

T3

T4

Unknown

Nodal status

$\begin{array}{ll}\text { N0 } & 139(39.6) \\ \text { N1 } & 112(31.8) \\ \text { N2 } & 61(17.3) \\ \text { N3 } & 39(11.0) \\ \text { Unknown } & 1(0.3)\end{array}$

Clinical stage

$$
\text { I }
$$

II

III

Unknown

Grading

G1

G2

G3

Unknown

Ki67

$\begin{array}{ll}<14 \% & 62(17.6) \\ \geq 14 \% & 256(72.7) \\ \text { Unknown } & 34(9.6)\end{array}$

HER2 status

Overexpressed (3+)

Amplified

Hormone receptor status

$\begin{array}{ll}\text { Negative } & 115(32.7) \\ \text { Positive } & 237(67.3) \\ \text { TP tumors } & 158(44.9) \\ \text { TP50 tumors } & 49(14.3)\end{array}$

Pts patients; TP triple positive tumors (HER2/ER/PgR-positive); TP50 TP patients with both ER and PgR staining in $50 \%$ or more tumor cells

\begin{tabular}{|c|c|c|}
\hline \multirow[t]{2}{*}{ Characteristics } & \multicolumn{2}{|l|}{$N(\%)$} \\
\hline & $\begin{array}{l}\text { Cohort A } \\
(352 \text { pts) }\end{array}$ & $\begin{array}{l}\text { Cohort B } \\
\text { (573 pts) }\end{array}$ \\
\hline \multicolumn{3}{|l|}{ Surgery } \\
\hline Conservative & $201(57.1)$ & $346(60.4)$ \\
\hline Radical & $151(42.9)$ & $227(39.6)$ \\
\hline \multicolumn{3}{|l|}{ Chemotherapy regimens } \\
\hline Anthracyclines-based & $174(49.4)$ & $92(16.1)$ \\
\hline Taxanes-based & $17(4.8)$ & $82(14.3)$ \\
\hline Anthracyclines and Taxanes-based & $70(19.9)$ & $374(65.3)$ \\
\hline $\begin{array}{l}\text { Non-anthracyclines and } \\
\text { Non-taxanes-based }\end{array}$ & $89(25.3)$ & $18(3.1)$ \\
\hline Unknown & $2(0.6)$ & $7(1.2)$ \\
\hline \multicolumn{3}{|l|}{ Cycles of chemotherapy } \\
\hline$<6$ & $95(27)$ & $84(14.7)$ \\
\hline$\geq 6$ & $255(72.4)$ & $483(84.3)$ \\
\hline Unknown & $2(0.6)$ & $6(1)$ \\
\hline \multicolumn{3}{|l|}{ Radiotherapy } \\
\hline Yes & $199(56.5)$ & $407(71)$ \\
\hline Not & $153(43.5)$ & $166(29)$ \\
\hline \multicolumn{3}{|l|}{ Hormonal therapy } \\
\hline Yes & $237(67.3)$ & $385(67.2)$ \\
\hline Not & $115(32.7)$ & $188(32.8)$ \\
\hline
\end{tabular}

Table 2 Main treatment characteristics in 925 patients

Pts patients

With regard to RR-3 year, the odds ratio (OR), and the $95 \%$ CI were estimated for each variable. A multivariate logistic regression model was developed using stepwise regression (forward selection). Enter limit and remove limit were $p=0.10$ and $p=0.15$, respectively. SPSS software (SPSS version 21.0, SPSS Inc., Chicago, Illinois, USA) was used for statistical evaluations.

\section{Results}

Patient characteristics and histopathological features

We identified 925 HER2-positive, consecutive, early breast cancer patients treated in ten Italian cancer centers over a 13-year time window.

Main baseline patients and tumor characteristics are reported in Table 1. Overall, HER2 was overexpressed $(3+)$ in $85.4 \%$ and HER2 gene was amplified in $14.6 \%$ of patients.

Among patients enrolled, 352 (38\%) received adjuvant chemotherapy without trastuzumab (cohort A) while 573 (62\%) patients received adjuvant chemotherapy followed by or combined with trastuzumab (cohort B). Median age, 
menopausal status, and mean tumor size were well-balanced between the two cohorts. Axillary lymph-nodes were positive in 212 patients $(60.1 \%)$ in the cohort $A$ and in 269 (47\%) patients in the cohort B. Among node-negative patients, there were $22 \mathrm{pT} 1 \mathrm{a} / \mathrm{b}$ and $70 \mathrm{pT} 1 \mathrm{c}$ tumors in the cohort A, and $52 \mathrm{pT} 1 \mathrm{a} / \mathrm{b}$ and $154 \mathrm{pT} 1 \mathrm{c}$ in the cohort B. Ki67 was $\geq 14$ in $72.7 \%$ of tumors in the cohort A and in $78.7 \%$ in the cohort B. Overall, 622 patients had ER and/ or PgR (HR)-positive tumors in the two cohorts, (67.3 and $67.2 \%$ in the cohort $\mathrm{A}$ and $\mathrm{B}$, respectively), and were prescribed endocrine adjuvant therapy at the end of chemotherapy. HR negative tumors had more frequently higher histologic grade $(p<0.0001)$ and larger tumor size $(p<0.0001)$ than HR-positive tumors. We identified 441 HER2/ER/PgR-positive (triple positive, TP) tumors, 158 in the cohort A, and 283 in the cohort B. TP tumors were more likely to present with more favorable histologic grade $(p<0.0001)$ and lower $\operatorname{Ki67}(p=0.008)$. Among TP tumors, we identified 163 tumors with both ER and PgR staining in $\geq 50 \%$ of tumor cells (TP50), 49 in the cohort $\mathrm{A}$, and 114 in the cohort B.

\section{Systemic treatment}

Data on adjuvant treatment are reported in Table 2.

On the whole population, $28.7 \%$ of the patients received anthracyclines-based, $10.7 \%$ taxane-based, $48 \%$ anthracycline-taxane-based, and $11.6 \%$ anthracyclinetaxane-free chemotherapy.

In the cohort $\mathrm{A}$, chemotherapy regimens were anthracycline-based in $49.4 \%$, taxane-based in $4.8 \%$, anthracycline-taxane-based in $19.9 \%$, anthracycline-taxane-free in $25.3 \%$ of the patients. In the cohort B, chemotherapy regimens were anthracycline-based in $16.1 \%$, taxanebased in $14.3 \%$, anthracycline-taxane-based in $65.3 \%$, anthracycline-taxane-free in $3.1 \%$ of the patients.

Two-hundred and fifty-five, and 483 patients (72.4 and $84.3 \%$ ) received $\geq 6$ cycles in the cohort $\mathrm{A}$ and $\mathrm{B}$, respectively.

In the cohort B, trastuzumab was given sequentially in $42 \%$ of the patients, and concomitantly with chemotherapy in $58 \%$ of the patients. The majority of patients received trastuzumab every 3 weeks $(94 \%)$. The median duration of trastuzumab treatment was 52 weeks (range, 1-104).

\section{Survival analyses}

The median follow-up of the entire population was 65 months (range 1-214), 102 months (range 2-214) in the cohort A, and 55 months (range 1-148) in the cohort B.

We observed 201 cases of disease recurrence, 136 in the cohort $\mathrm{A}$, and 65 in the cohort $\mathrm{B}$.
The RR-3 year for patients in the cohort A was $18.5 \%$, and in the cohort B it was $7.8 \%(p<0.0001)$.

Kaplan-Meier curves showed that the 5-year RFS in the cohort A was $71 \%$, while it was $88.6 \%$ in the cohort B $(p<0.0001)$ (Fig. 1a, b). The 5-year OS was 88.4 and $96 \%$ in the cohort A and B respectively, while the 10-year OS was 72.3 and $93.3 \%$ in the cohort $\mathrm{A}$ and $\mathrm{B}$ $(p<0.0001)$. The 5-year BCSS was 89.2 and $96.2 \%$ in the cohort A and B, the 10-year BCSS was 73.3 and $93.5 \%$ in the cohort $\mathrm{A}$ and $\mathrm{B}(p<0.0001)$.

The higher hazard rate of recurrence was in the first 5 years in both cohorts (Fig. 2). In multivariate analysis, the whole population factors related to relapse were younger age, advanced stage at diagnosis, absence of hormonal treatment and of trastuzumab therapy (Table 3).

Among node-negative patients, the RR-3 year was $14.4 \%$ in cohort $\mathrm{A}$ and $3.6 \%$ in cohort $\mathrm{B}(p<0.0001)$, whereas in node-positive group these values were 20.6 and $12.3 \%$, respectively ( $p=0.02$ ). Kaplan-Meier curves showed that, in node-negative subgroup, the 5-year RFS was 76.2\% in the cohort A and $93.9 \%$ in the cohort B $(p<0.0001)$; the 5-year OS was 90.5 and $97.6 \%$ in the cohort A and B, respectively $(p=0.0002)$. In node-positive subgroup, the 5-year RFS was 68.1 and $82.8 \%$ in the cohort $\mathrm{A}$ and $\mathrm{B}$, respectively $(p=0.0003)$; the 5-year OS was $87.5 \%$ in the cohort $\mathrm{A}$ and $94.3 \%$ in the cohort B $(p=0.009)$.

Overall, HR-negative tumors had a trend toward a worse RR-3 year than HR positive tumors (15.2 and $10.5 \%$, respectively) $(p=0.05)$. The RR-3 year was 16.2 and $6.3 \%(p<0.0001)$ in patients with HR-positive tumors (ER and/or PgR positive) in the cohort A and B, respectively, whereas it was 22.8 and $10.7 \%$ in patients with HRnegative tumors $(p=0.007)$ in the cohort $\mathrm{A}$ and $\mathrm{B}$, respectively (Fig. 4). Overall, HR-negative disease had a trend toward a worse 5-year RFS outcome than HR-positive disease, but the difference was not statistically significant $(p=0.63)$. The 5-year RFS of patients with HRnegative tumors was $70.2 \%$ in the cohort $\mathrm{A}$, and $86.1 \%$ in the cohort B $(p=0.002)$. The 5-year RFS of patients with HR-positive tumors was $71.7 \%$ in the cohort A, while it was $89.7 \%$ in the cohort B ( $p<0.0001)$ (Fig. 3a). Overall, 5-year OS was $90.9 \%$ in the HR-negative group and $93.8 \%$ in the HR-positive group ( $p=0.36$ ). Among HRpositive patients, the 5-year OS was 90.7 and $96.1 \%$ in the cohort A and B, respectively ( $p=0.006)$, while for HRnegative patients it was 84.3 and $95.8 \%$ in the cohort $\mathrm{A}$ and $\mathrm{B}(p=0.002)$, respectively (Fig. 3b).

In the $441 \mathrm{TP}$ patients, the RR-3 year was $15 \%$ in cohort A and $6.4 \%$ in cohort B $(p=0.005)$ (Fig. 4). The 5-years RFS was $71.7 \%$ in the cohort A and $91 \%$ in the cohort B $(p<0.0001)$, while the 5-year OS was $92.1 \%$ in the cohort A and $96.6 \%$ in the cohort B $(p=0.005)$. Conversely, in the small subset of TP50 patients, the RR- 
Fig. 1 a Five-year relapse-free survival in the overall population. b Five-year overall survival in the overall population

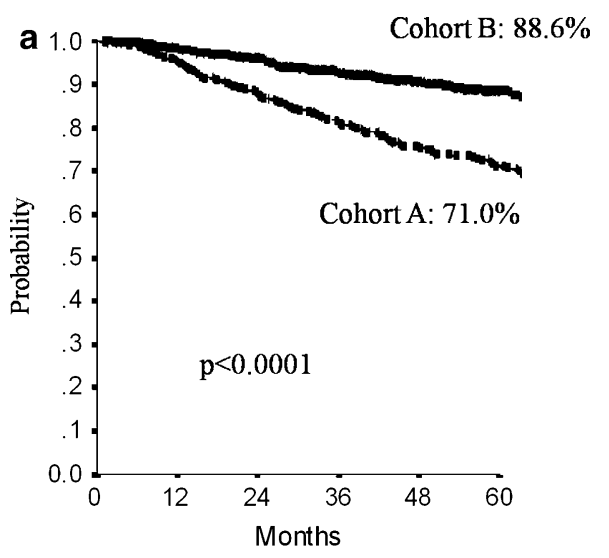

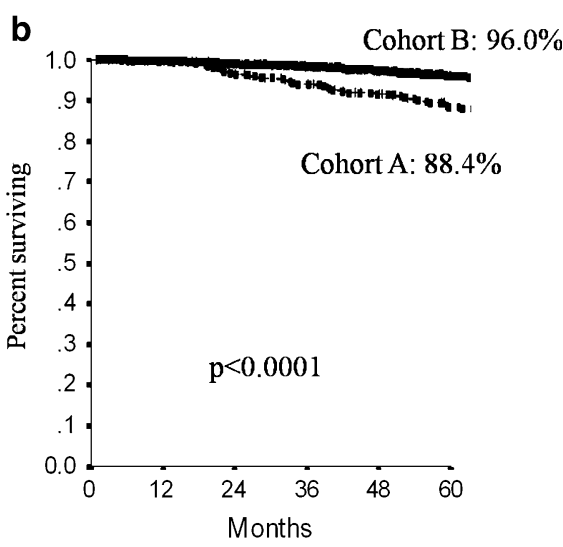

3 year was $6.2 \%$ in the cohort A and $5.4 \%$ in the cohort B $(p=0.84)$ (Fig. 4). The 5-years RFS was $89.7 \%$ in the cohort $\mathrm{A}$ and $92.3 \%$ in the cohort $\mathrm{B}(p=0.27)$, and the

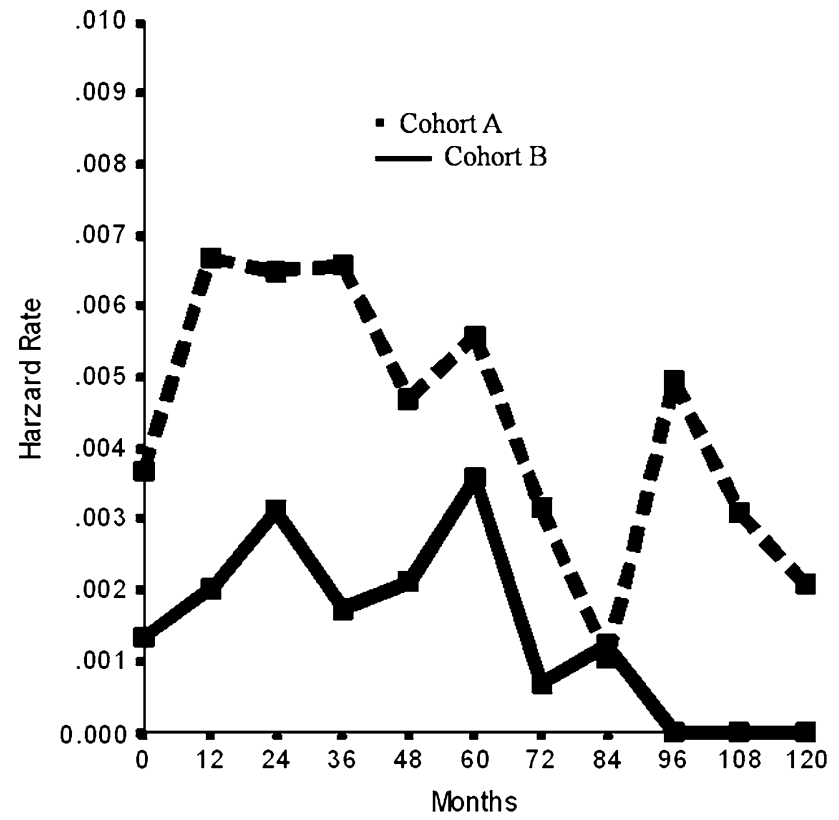

5-year OS was $95.7 \%$ in the cohort A and $94.9 \%$ in the cohort B $(p=0.37)$.

Twenty out of 59 patients with small (pT1a/b), nodenegative tumors received adjuvant chemotherapy without trastuzumab (cohort A), and 39 received trastuzumab (cohort B). The RR-3 year for patients with pT1a/b tumors was $20 \%$ in the cohort $\mathrm{A}$ and $5.1 \%$ in the cohort $\mathrm{B}$ ( $p=0.17$ ). In pT1c node-negative tumors, we analyzed 70 patients in the cohort A and 154 in the cohort B. The RR3 year was $10 \%$ in the cohort A and $4 \%$ in the cohort B $(p=0.08)$. In this subgroup, the 5-year RFS was $81.7 \%$ in the cohort $\mathrm{A}$ and $92.5 \%$ in the cohort $\mathrm{B}(p=0.003)$, while the 5-year OS was $84.3 \%$ in the cohort $\mathrm{A}$ and $95.8 \%$ in the cohort $\mathrm{B}(p=0.002)$.

When analyzing only patients who had received homogeneous adjuvant treatments with anthracyclines combined with or followed by taxanes, namely 66 patients in the cohort A and 317 patients in the cohort B, the RR3 year was $22.7 \%$ in cohort $\mathrm{A}$ and $7.9 \%$ in the cohort B $(p<0.0001)$; the 5-year RFS was $67.0 \%$ in the cohort A and $87.2 \%$ in the cohort B $(p<0.0001)$, while the 5-year OS was $86.4 \%$ in the cohort A and $95.7 \%$ in the cohort B ( $p=0.006)$, confirming the results of the overall population.

Fig. 2 Relapse rate in the overall population

Table 3 Multivariate analysis in 925 patients for relapse-free survival (RFS) and 3-years relapse-rate $(\mathrm{RR} 3 \mathrm{y})$

$C I$ confidence interval; $H R$ hazard ratio; $O R$ odds ratio

\begin{tabular}{|c|c|c|c|c|}
\hline \multirow[t]{2}{*}{ Factors } & \multicolumn{2}{|l|}{ RFS } & \multicolumn{2}{|l|}{ RR3y } \\
\hline & HR (95\%CI) & $p$ value & OR (95\%CI) & $p$ value \\
\hline \multicolumn{5}{|c|}{ Adjuvant hormonal therapy } \\
\hline No versus Yes & $1.72(1.26-2.37)$ & 0.001 & $2.54(1.56-4.13)$ & $<0.0001$ \\
\hline \multicolumn{5}{|l|}{ Stage } \\
\hline III versus I-II & $2.82(2.04-3.89)$ & $<0.0001$ & $3.55(2.17-5.81)$ & $<0.0001$ \\
\hline \multicolumn{5}{|c|}{ Adjuvant trastuzumab } \\
\hline No versus Yes & $2.27(1.62-3.19)$ & $<0.0001$ & $2.33(1.44-3.78)$ & 0.001 \\
\hline \multicolumn{5}{|l|}{ Age at diagnosis } \\
\hline$<55$ versus $>55$ & $1.53(1.11-2.12)$ & 0.01 & - & - \\
\hline
\end{tabular}


Fig. 3 a Five-year relapse-free survival according to hormonal receptor (HR) status. b Fiveyear overall survival according to hormonal receptor (HR) status
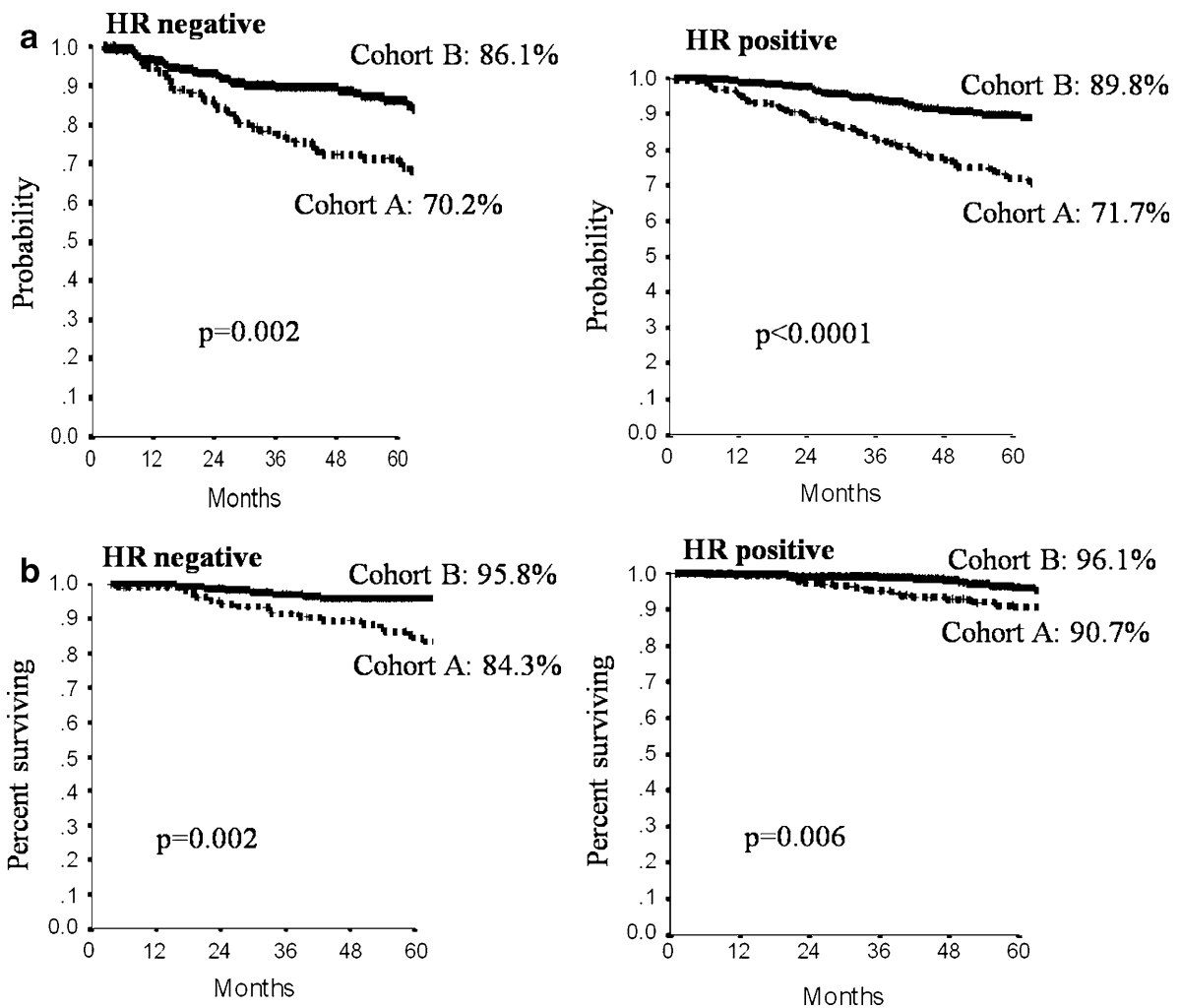

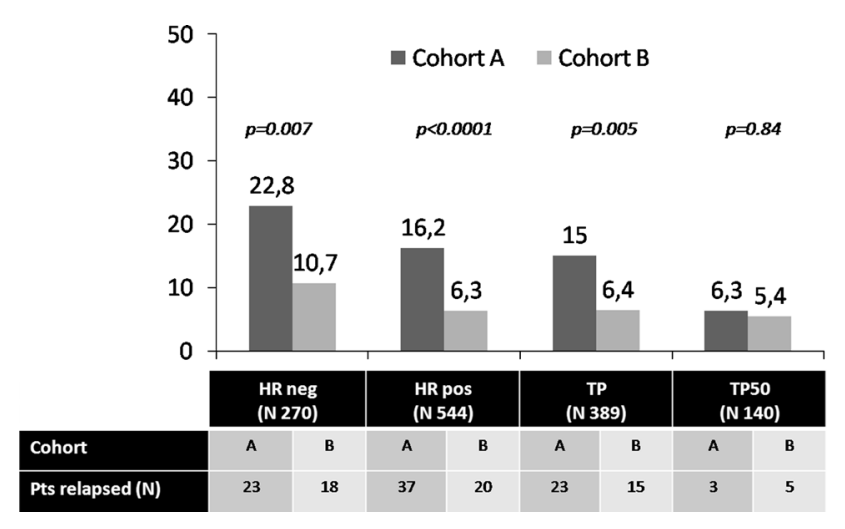

Fig. 4 Three-year relapse rate according to hormonal receptor (HR) status. HR hormonal receptor; neg negative; pos positive; TP triple positive; $N$ number; $p t s$ patients. This analysis was performed only for patients with adequate follow-up

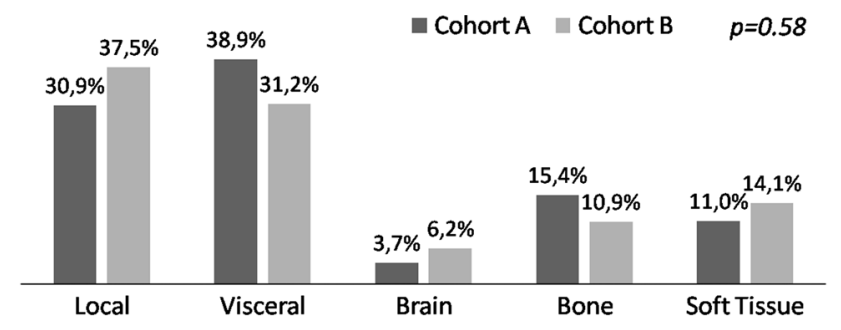

Fig. 5 Incidence of first recurrence according to adjuvant treatment in 201 patients recurred (136 in the cohort A, 65 in the cohort B)
In regard to first site of recurrence, even non-significant, we observed in the cohort A more visceral and bone recurrences and less local and soft tissue recurrences than in trastuzumab-treated patients, who conversely experienced a non-significant trend toward a higher incidence of brain recurrence, which was $3.7 \%$ in the cohort $\mathrm{A}$ and $6.3 \%$ in the cohort B $(p=0.65)$ (Fig. 5). Overall, TP tumors were more likely to recur in soft tissues with respect to non-TP tumors $(p=0.03)$ (16 out of 24 soft tissue recurrences, 12 in the cohort A and 4 in the cohort B), and developed 3 out of 9 brain metastases, but no conclusion can be drawn due to the small sample size. There was no difference with regard to the sites of recurrence in TP50 tumors, but numbers are very low.

\section{Discussion}

Our results confirm the relevant impact of adjuvant trastuzumab in the outcome of patients with HER2-positive tumors treated in real-world practice.

Overall, the RR-3 year observed was significantly more favorable in the trastuzumab cohort $(7.8 \%)$ compared to that of patients treated with chemotherapy without trastuzumab $(18.5 \%), \quad(p<0.0001)$. Kaplan-Meier curves confirmed a more favorable 5-year RFS $(88.6 \%$ vs $71 \%$, $p<0.0001$ ), 5-year OS (96\% vs $88.4 \%, p<0.0001$ ), and 
5-year BCSS $(96.2 \%$ vs $89.2 \%, p<0.0001)$ in the cohort $\mathrm{B}$, thus corroborating the advantage of adding trastuzumab to standard adjuvant treatments.

Even if trastuzumab benefit was confirmed in a subgroup analysis including a more homogeneous subset receiving sequential regimens with anthracyclines and taxanes, it is not possible to draw any conclusion on the weight of the type of adjuvant chemotherapy regimens delivered, because the number of patients were unbalanced in the two cohorts.

More patients with lymph node-positive tumors were recruited in the cohort $A$ than in the cohort B (60 and $47 \%$ ), possibly reflecting the recent introduction of more organized screening programs. The advantage of trastuzumab administration was observed in node-positive and node-negative subgroups, both showing more favorable survival rates in the cohort $B$. In the node-positive subgroup, the advantage seems more pronounced at a longer follow-up period, since at the first analysis (RR-3 year) the benefit appeared less noticeable $(p=0.02)$, while the 5 -year RFS showed a higher difference between the two cohorts $(p=0.003)$.

Trastuzumab benefit in our population was observed in both HR-negative and HR-positive tumors (Fig. 3a), with a trend of HR-negative tumors toward a worse outcome. This is consistent with results of another retrospective analysis of $897 \mathrm{TP}$ patients treated with chemotherapy and trastuzumab between 1988 and 2009, showing a clear advantage in DFS/OS for patients who received adjuvant endocrine treatment [13].

In the subset of TP tumors, the RR-3 year was significantly lower in the cohort B $(6.4 \%)$ than in the cohort A $(15 \%)(p=0.005)$, and at a longer follow-up, the trastuzumab benefit became more pronounced, since the 5-year RFS was $71 \%$ in the cohort A and $88.6 \%$ in the cohort B $(p<0.0001)$, and the 5-year OS was $89.2 \%$ and $96.2 \%$ in the cohort A and $\mathrm{B}$, respectively $(p<0.0001)$. This confirms that adding trastuzumab to chemotherapy and hormonal therapy in TP patients is highly beneficial, even if the advantage seems more prominent when we consider a longer follow-up, in order to take into account late recurrences. However, the relatively limited number of patients included in TP subgroup, and the low number of events, refrains us from drawing any firm conclusion and encourage further studies on similarly characterized patients.

In our series, the RR-3 year in TP50 tumors was $6.2 \%$ in the cohort $\mathrm{A}$ and $5.4 \%$ in the cohort $\mathrm{B}$ $(p=0.99)$, suggesting a lack of trastuzumab benefit, possibly related to an intrinsically more favorable prognosis in patients with tumors expressing high levels of HR, or to very late recurrences not detected by the current analysis, but we cannot draw any conclusion because of the small sample size, the unbalanced number of patients between the two cohorts and the extremely low number of events.

Our results confirm a higher recurrence risk in the cohort A even in the subset of small-size node-negative tumors, further substantiating previous literature data [14-19].

In the present analysis, we found some suggestions of a higher incidence of brain metastases as the first site of recurrence in the cohort $\mathrm{B}$, even if it is not statistically extent, but results are impaired by the low number of events. The increased rate of brain metastases in patients treated with trastuzumab is still a matter of discussion. Results from two metanalyses of randomized trials have shown a significantly higher incidence of brain metastases in the arms treated with trastuzumab [20, 21], possibly due to the longer survival of trastuzumab-treated patients. Conversely, a retrospective evaluation of the HERA trial did not confirm an increased risk of developing brain metastases in the trastuzumab arm [22]. Our data suggested a higher incidence of soft tissue recurrences in TP tumors, according to the different pattern of metastatic spread related to HR status [23]; however, our results are largely impaired by the low number of events.

Prior to the availability of adjuvant trastuzumab, the major factor limiting patient survival was the progression of systemic disease, with the HER2 subtype representing an unfavorable subgroup. Following the introduction of trastuzumab, the prognosis of patients with HER2-positive tumors showed significant improvements, confirmed by a recent meta-analysis by the Cochrane group, including six adjuvant and two neoadjuvant randomized trials [24].

Trastuzumab benefit in the adjuvant setting is also described by some retrospective analyses, but number of patients is usually low, pathological and clinical parameters heterogeneous, and follow-up periods frequently short. The GHEA study, recruiting 1.002 patients treated with sequential adjuvant trastuzumab, reported data similar to those of the HERA trial, but the median follow-up was 32 months [11]. Recently, a population-based study examined diverse consecutive series of breast cancers including a very small subset of HER2-positive subtypes, before trastuzumab registration, and patients not treated with any HER2 targeted therapy showed the worst outcome in terms of OS and DFS of the entire HER2-positive population [25]. Another population-based study of Canadian patients, including HR-positive, a small subset of 94 HER2-positive, and triple negative tumors in the "trastuzumab era", and so treated with trastuzumab whenever indicated, showed that patients with HER2-positive and HR-positive tumors had similar outcomes, while triple negative patients had the worst outcome, confirming the prognostic impact of trastuzumab administration [26]. A retrospective evaluation of HER2-positive early breast cancer treated with or without trastuzumab in routine 
clinical practice between 2006 and 2008 was carried out by Palmieri et al., confirming that trastuzumab-untreated patients had an increased risk of recurrence, but analysis was conducted on a low number of patients [27]. Recent data on 1,134 patients with HER2-positive early breast cancer from a German clinical cancer registry showed that the best OS was found in HER2-positive/HR-positive patients receiving trastuzumab plus chemotherapy plus endocrine therapy, compared to the non-trastuzumab group. In multivariable analysis, HER2-positive patients treated with chemotherapy and/or endocrine therapy without trastuzumab had a worse OS than the control groups [28].

Certainly, the present study presents some substantial limitations. Among the main weaknesses, the retrospective design makes this study prone to confounding and bias. We also have to consider the heterogeneity of adjuvant treatments delivered, and the different follow-up length for the two cohorts; indeed, some late recurrences might have been possibly missed in the youngest cohort, namely, cohort B. In regard to the chemotherapy regimen delivered, a minority of patients received sequential anthracyclines and taxanes in the cohort $\mathrm{A}$, and this could be another limiting factor, since the variability in chemotherapy schemes may have influenced results, even if the analysis in the subgroup of patients treated with anthracycline and taxane-based regimens seemed to confirm the findings from the entire patient population. Moreover, though interesting and suggestive of hypotheses warranting further investigations, the apparent lack of effect of trastuzumab observed in patients with tumors with very high levels of HR (TP50) should be cautiously considered, particularly due to relatively low number of patients per each subgroup. In addition, the low number of total recurrence or death events should be acknowledged as a general limiting factor for data analyses.

Despite these limitations, our study has some important strengths. Results from this analysis stems from a large series of patients, all treated according to standard guidelines and with a long length of follow-up which enabled us to capture the greater part of late recurrences also.

To sum up, our results confirm the benefit of trastuzumab administration on patients treated outside of clinical trials in quite all the subtypes of HER2-positive tumors, and make the argument to perform further trials in molecularly defined subgroups. In fact, data on the effect of trastuzumab in routine setting, and particularly in HER2positive subgroups, are still scarce, deriving usually from low number of patients and with frequently short follow-up periods. Moreover, results herein presented, particularly in specific subsets such as the TP tumors and in tumors with high HR expression, require further evaluation. In these subsets, hormonal manipulations are the mainstay of treatment, whereas the benefit coming from chemotherapy is of a lower extent compared to other intrinsic subtypes. Likewise, in these tumors the impact of adjuvant trastuzumab on long-term outcomes is not so well-defined. From a biological standpoint, we might speculate that in an oncogenic background dominated by hormone-related stimuli, HER2 signaling might not be essential for cancer cells. To test this hypothesis, we are now performing a wider observational retrospective study in order to achieve a more exact picture on trastuzumab benefit in TP and TP50 tumors.

Acknowledgments We thank Anna Maria Edlisca for technical assistance.

Conflict of interests The authors declare that they have no conflict of interest.

Ethical Standards This study was conducted in accordance with the Good Clinical Practice guidelines, the Declaration of Helsinki, and each country's laws and regulations.

Open Access This article is distributed under the terms of the Creative Commons Attribution Noncommercial License which permits any noncommercial use, distribution, and reproduction in any medium, provided the original author(s) and the source are credited.

\section{References}

1. Sotiriou C, Wirapati P, Loi S, Harris A, Fox S, Smeds J, Nordgren H, Farmer P, Praz V, Haibe-Kains B, Desmedt C, Larsimont D, Cardoso F, Peterse H, Nuyten D, Buyse M, Van de Vijver MJ, Bergh J, Piccart M, Delorenzi M (2006) Gene expression profiling in breast cancer: understanding the molecular basis of histologic grade to improve prognosis. J Natl Cancer Inst 98(4):262-272

2. Slamon DJ, Clark GM, Wong SG, Levin WJ, Ullrich A, McGuire WL (1987) Human breast cancer: correlation of relapse and survival with amplification of the HER-2/neu oncogene. Science 235(4785):177-182

3. Slamon DJ, Leyland-Jones B, Shak S, Fuchs H, Paton V, Bajamonde A, Fleming T, Eiermann W, Wolter J, Pegram M, Baselga J, Norton L (2001) Use of chemotherapy plus a monoclonal antibody against HER2 for metastatic breast cancer that overexpresses HER2. N Engl J Med 344(11):783-792

4. Piccart-Gebhart MJ, Procter M, Leyland-Jones B, Goldhirsch A, Untch M, Smith I, Gianni L, Baselga J, Bell R, Jackisch C, Cameron D, Dowsett M, Barrios CH, Steger G, Huang CS, Andersson M, Inbar M, Lichinitser M, Láng I, Nitz U, Iwata $H$, Thomssen C, Lohrisch C, Suter TM, Rüschoff J, Suto T, Greatorex V, Ward C, Straehle C, McFadden E, Dolci, Gelber RD, Herceptin Adjuvant (HERA) Trial Study Team (2005) Trastuzumab after adjuvant chemotherapy in HER2-positive breast cancer. N Engl J Med 353(16):1659-1672

5. Gianni L, Dafni U, Gelber RD, Azambuja E, Muehlbauer S, Goldhirsch A, Untch M, Smith I, Baselga J, Jackisch C, Cameron D, Mano M, Pedrini JL, Veronesi A, Mendiola C, Pluzanska A, Semiglazov V, Vrdoljak E, Eckart MJ, Shen Z, Skiadopoulos G, Procter M, Pritchard KI, Piccart-Gebhart MJ, Bell R, Herceptin Adjuvant (HERA) Trial Study Team (2011) Treatment with trastuzumab for 1 year after adjuvant chemotherapy in patients 
with HER2-positive early breast cancer: a 4-year follow-up of a randomised controlled trial. Lancet Oncol 12(3):236-244

6. Romond EH, Perez EA, Bryant J, Suman VJ, Geyer CE Jr, Davidson NE, Tan-Chiu E, Martino S, Paik S, Kaufman PA, Swain SM, Pisansky TM, Fehrenbacher L, Kutteh LA, Vogel VG, Visscher DW, Yothers G, Jenkins RB, Brown AM, Dakhil SR, Mamounas EP, Lingle WL, Klein PM, Ingle JN, Wolmark N (2005) Trastuzumab plus adjuvant chemotherapy for operable HER2-positive breast cancer. N Engl J Med 353(16):1673-1684

7. Slamon D, Eiermann W, Robert N, Pienkowski T, Martin M, Press M, Mackey J, Glaspy J, Chan A, Pawlicki M, Pinter T, Valero V, Liu MC, Sauter G, von Minckwitz G, Visco F, Bee V, Buyse M, Bendahmane B, Tabah-Fisch I, Lindsay MA, Riva A, Crown J, Breast Cancer International Research Group (2011) Adjuvant trastuzumab in HER2-positive breast cancer. N Engl J Med 365(14):1273-1283

8. Joensuu H, Bono P, Kataja V, Alanko T, Kokko R, Asola R, Utriainen T, Turpeenniemi-Hujanen T, Jyrkkiö S, Möykkynen K, Helle L, Ingalsuo S, Pajunen M, Huusko M, Salminen T, Auvinen $\mathrm{P}$, Leinonen H, Leinonen M, Isola J, Kellokumpu-Lehtinen PL (2009) Fluorouracil, epirubicin, and cyclophosphamide with either docetaxel or vinorelbine, with or without trastuzumab, as adjuvant treatments of breast cancer: final results of the FinHer trial. J Clin Oncol 27(34):5685-5692

9. Perez EA, Suman VJ, Davidson NE, Gralow JR, Kaufman PA, Visscher DW, Chen B, Ingle JN, Dakhil SR, Zujewski J, MorenoAspitia A, Pisansky TM, Jenkins RB (2011) Sequential versus concurrent trastuzumab in adjuvant chemotherapy for breast cancer. J Clin Oncol 29(34):4491-4497

10. Viani GA, Afonso SL, Stefano EJ, De Fendi LI, Soares FV (2007) Adjuvant trastuzumab in the treatment of HER-2-positive early breast cancer: a meta-analysis of published randomized trials. BMC Cancer 7:153

11. Campiglio M, Bufalino R, Sasso M, Ferri E, Casalini P, Adamo V, Fabi A, Aiello R, Riccardi F, Valle E, Scotti V, Tabaro G, Giuffrida D, Tarenzi E, Bologna A, Mustacchi G, Bianchi F, Balsari A, Ménard S, Tagliabue E (2013) Effect of adjuvant trastuzumab treatment in conventional clinical setting: an observational retrospective multicenter Italian study. Breast Cancer Res Treat 141(1):101-110

12. Pocock SJ, Clayton TC, Altman DG (2002) Survival plots of time-to-event outcomes in clinical trials: good practice and pitfalls. Lancet 359(9318):1686-1689

13. Hayashi N, Niikura N, Yamauchi H, Nakamura S, Ueno NT (2013) Adding hormonal therapy to chemotherapy and trastuzumab improves prognosis in patients with hormone receptorpositive and human epidermal growth factor receptor 2-positive primary breast cancer. Breast Cancer Res Treat 137(2):523-531

14. Gamucci T, Vaccaro A, Ciancola F, Pizzuti L, Sperduti I, Moscetti L, Longo F, Fabbri MA, Giampaolo MA, Mentuccia L, Di Lauro L, Vici P (2013) Recurrence risk in small, node-negative, early breast cancer: a multicenter retrospective analysis. J Cancer Res Clin Oncol 139(5):853-860

15. Rodrigues MJ, Wassermann J, Albiges L, Brain E, Delaloge S, Stevens D, Guinebretière JM, Mathieu MC, Kirova Y, Guillot E, VincentSalomon A, Cottu PH (2010) Trastuzumab treatment in tlab, nodenegative, human epidermal growth factor receptor 2-overexpressing breast carcinomas. J Clin Oncol 28(28):e541-542

16. McArthur HL, Mahoney KM, Morris PG, Patil S, Jacks LM, Howard J, Norton L, Hudis CA (2011) Adjuvant trastuzumab with chemotherapy is effective in women with small, nodenegative, HER2-positive breast cancer. Cancer 117(24): $5461-5468$
17. Horio A, Fujita T, Hayashi H, Hattori M, Kondou N, Yamada M, Adachi E, Ushio A, Gondou N, Sueta A, Yatabe Y, Iwata H (2012) High recurrence risk and use of adjuvant trastuzumab in patients with small, HER2-positive, node-negative breast cancers. Int J Clin Oncol 17(2):131-136

18. Park YH, Kim ST, Cho EY, Choi YL, Ok ON, Baek HJ, Lee JE, Nam SJ, Yang JH, Park W, Choi DH, Huh SJ, Ahn JS, Im YH (2010) A risk stratification by hormonal receptors (ER, PgR) and HER-2 status in small $(<$ or $=1 \mathrm{~cm})$ invasive breast cancer: who might be possible candidates for adjuvant treatment? Breast Cancer Res Treat 119(3):653-661

19. Curigliano G, Viale G, Bagnardi V, Fumagalli L, Locatelli M, Rotmensz N, Ghisini R, Colleoni M, Munzone E, Veronesi P, Zurrida S, Nolè F, Goldhirsch A (2009) Clinical relevance of HER2 overexpression/amplification in patients with small tumor size and node-negative breast cancer. J Clin Oncol 27(34):56935699

20. Bria E, Cuppone F, Fornier M, Nisticò C, Carlini P, Milella M, Sperduti I, Terzoli E, Cognetti F, Giannarelli D (2008) Cardiotoxicity and incidence of brain metastases after adjuvant trastuzumab for early breast cancer: the dark side of the moon? A meta-analysis of the randomized trials. Breast Cancer Res Treat 109(2):231-239

21. Olson EM, Abdel-Rasoul M, Maly J, Wu CS, Lin NU, Shapiro CL (2013) Incidence and risk of central nervous system metastases as site of first recurrence in patients with HER2-positive breast cancer treated with adjuvant trastuzumab. Ann Oncol 24(6):1526-1533

22. Pestalozzi BC, Holmes E, de Azambuja E, Metzger-Filho O, Hogge L, Scullion M, Láng I, Wardley A, Lichinitser M, Sanchez RI, Müller V, Dodwell D, Gelber RD, Piccart-Gebhart MJ, Cameron D (2013) CNS relapses in patients with HER2-positive early breast cancer who have and have not received adjuvant trastuzumab: a retrospective substudy of the HERA trial (BIG 1-01). Lancet Oncol 14(3):244-248

23. Park YH, Lee S, Cho EY, Choi YL, Lee JE, Nam SJ, Yang JH, Ahn JS, Im YH (2010) Patterns of relapse and metastatic spread in HER2-overexpressing breast cancer according to estrogen receptor status. Cancer Chemother Pharmacol 66(3):507-516

24. Moja L, Tagliabue L, Balduzzi S, Parmelli E, Pistotti V, Guarneri V, D'Amico R (2012) Trastuzumab containing regimens for early breast cancer. Cochrane Database Syst Rev 4:CD006243. doi:10. 1002/14651858.CD006243.pub2

25. Purdie CA, Baker L, Ashfield A, Chatterjee S, Jordan LB, Quinlan P, Adamson DJ, Dewar JA, Thompson AM (2010) Increased mortality in HER2 positive, oestrogen receptor positive invasive breast cancer: a population-based study. $\mathrm{Br} \mathrm{J}$ Cancer 103(4):475-481

26. Zurawska U, Baribeau DA, Giilck S, Victor C, Gandhi S, Florescu A, Verma S (2013) Outcomes of HER2-positive early-stage breast cancer in the trastuzumab era: a population-based study of Canadian patients. Curr Oncol 20(6):e539-545

27. Palmieri C, Shah D, Krell J, Gojis O, Hogben K, Riddle P, Ahmad R, Tat T, Fox K, Porter A, Mahmoud S, Kirschke S, Shousha S, Gudi M, Coombes RC, Leonard R, Cleator S (2011) Management and outcome of HER2-positive early breast cancer treated with or without trastuzumab in the adjuvant trastuzumab era. Clin Breast Cancer 11(2):93-102

28. Inwald EC, Ortmann O, Zeman F, Koller M, Hofstädter F, Gerstenhauer M, Klinkhammer-Schalke M (2014) Guideline concordant therapy prolongs survival in HER2-positive breast cancer patients: results from a large population-based cohort of a cancer registry. Biomed Res Int. doi:10.1155/2014/137304 